\title{
ERGODIC THEOREMS OF WEAK MIXING TYPE
}

\author{
LEE K. JONES AND MICHAEL LIN ${ }^{1}$
}

Abstract. Given a linear contraction $T$ on a Banach space $X$ and $x \in X$, the convergence

$$
\forall x^{*} \in X^{*} N^{-1} \sum_{i=1}^{N}\left|\left\langle x^{*}, T^{i} x\right\rangle\right| \underset{N \rightarrow \infty}{\longrightarrow} 0
$$

is shown to be equivalent to the convergence

$$
\sup _{\left\|x^{*}\right\|<1} N^{-1} \sum_{j=1}^{N}\left|\left\langle x^{*}, T^{k_{j}} x\right\rangle\right| \rightarrow 0
$$

for every subsequence with $k_{j} / j$ bounded. A sufficient condition is that, for some $\left\{n_{i}\right\}, T^{n_{i}} x \rightarrow 0$ weakly.

The following theorem gives a characterization of weak mixing for operators in general Banach spaces, generalizing the well-known results for measure preserving transformations. For more details see [2].

THEOREM. Let $T$ be a linear operator on a Banach space with $\sup _{n} \geqslant 0\left\|T^{n}\right\|$ $<\infty$, and let $x \in X$. Then the following conditions are equivalent.

(i) For every $x^{*} \in X^{*}$ we have $\lim _{N \rightarrow \infty} N^{-1} \sum_{j=1}^{N}\left|\left\langle x^{*}, T^{j} x\right\rangle\right|=0$.

$$
\sup _{\left\|x^{*}\right\| \leqslant 1} N^{-1} \sum_{j=1}^{N}\left|\left\langle x^{*}, T^{j} x\right\rangle\right| \underset{N \rightarrow \infty}{\longrightarrow} 0 .
$$

(iii) For every subsequence $\left\{k_{j}\right\}$ with positive lower density (i.e., sup $k_{j} / j<\infty$ ) we have $\lim _{N \rightarrow \infty}\left\|N^{-1} \sum_{j=1}^{N} T^{k_{j}} x\right\|=0$.

(iv) For every subsequence $\left\{k_{j}\right\}$ with positive lower density,

$$
\sup _{\left\|x^{*}\right\| \leqslant 1} N^{-1} \sum_{j=1}^{N}\left|\left\langle x^{*}, T^{k_{j}} x\right\rangle\right| \underset{N \rightarrow \infty}{\longrightarrow} 0 .
$$

Proof. (i) $\Rightarrow$ (ii): We first assume $\|T\| \leqslant 1$. Let $B=\left\{x^{*} \in X^{*}:\left\|x^{*}\right\|\right.$ $\leqslant 1$ \}. $B$ is a compact Hausdorff space in the $w^{*}$-topology, and $T^{*}(B) \subset B$. Since $T^{*}$ is $w^{*}$-continuous on $B$, the operator $A f\left(x^{*}\right)=f\left(T^{*} x^{*}\right)$ is a contraction of $C(B)$. For $g\left(x^{*}\right)=\left|\left\langle x^{*}, x\right\rangle\right|$, we have by (i) that $N^{-1} \sum_{j=1}^{N} A^{j} g$ $\rightarrow 0$ pointwise. Hence for $\mu \in C(B)^{*}$ satisfying $A^{*} \mu={ }^{\prime} \mu$ we have $\langle\mu, g\rangle=0$,

Received by the editors March 21, 1975.

AMS (MOS) subject classifications (1970). Primary 47A35; Secondary 28A65, $60 \mathrm{~J} 05$.

Key words and phrases. Ergodic theorems, weak mixing.

${ }^{1}$ Research supported in part by NSF grant GP 34118. 
and $g \in \overline{(I-A) C(B)}$, yielding $\left\|N^{-1} \sum_{j=1}^{N} A^{j} g\right\| \rightarrow 0$ and (ii) holds. Now let $\sup _{n \geqslant 0}\left\|T^{n}\right\|=M, 1<M<\infty$. We define an equivalent norm in $X$ by $\|y\|=\sup \left\|T^{n} y\right\|$. Then $\|T\| \leqslant 1$, and since (i) is not altered, we have, by the previous proof and using $\left\|x^{*}\right\| \leqslant\left\|x^{*}\right\|$, that

$$
\sup _{\left\|x^{*}\right\| \leqslant 1} N^{-1} \sum_{j=1}^{N}\left|\left\langle x^{*}, T^{j} x\right\rangle\right| \leqslant \sup _{\left\|x^{*}\right\| \leqslant 1} N^{-1} \sum_{j=1}^{N}\left|\left\langle x^{*}, T^{j} x\right\rangle\right| \underset{N \rightarrow \infty}{\longrightarrow} 0 .
$$

(ii) $\Rightarrow$ (iv): Let $k_{j} / j \leqslant a<\infty$. Then

$$
\begin{aligned}
\sup _{\left\|x^{*}\right\| \leqslant 1} N^{-1} \sum_{j=1}^{N}\left|\left\langle x^{*}, T^{k_{j}} x\right\rangle\right| & \leqslant \sup _{\left\|x^{*}\right\| \leqslant 1} N^{-1} \sum_{i=1}^{k_{N}}\left|\left\langle x^{*}, T^{i} x\right\rangle\right| \\
& \leqslant a \sup _{\left\|x^{*}\right\| \leqslant 1}\left(k_{N}\right)^{-1} \sum_{i=1}^{k_{N}}\left|\left\langle x^{*}, T^{i} x\right\rangle\right| \underset{N \rightarrow \infty}{\longrightarrow} 0 .
\end{aligned}
$$

(iv) $\Rightarrow$ (iii) is immediate.

(iii) $\Rightarrow$ (i) is proved (easily) in the last paragraph of [2, Theorem IV].

Proposition. Let $\sup \left\|T^{n}\right\|<\infty$ and $x \in X$. If for some subsequence $\left\{n_{i}\right\}$, $T^{n_{i}} x \rightarrow 0$ weakly, then (ii) holds.

Proof. When $\|T\| \leqslant 1$, let $A$ on $C(B)$ and $g$ be as in the proof of the Theorem. Then $A^{n_{i}} g \rightarrow 0$ pointwise, and $\langle\mu, g\rangle=0$ for every $\mu \in C(B)^{*}$ satisfying $A^{*} \mu=\mu$. The remainder of the proof is exactly the same as before.

REMARKs. 1. If $X^{*}$ is separable, then any of the conditions of the Theorem implies the existence of a subsequence $\left\{n_{i}\right\}$ such that $T^{n_{i}} x \rightarrow 0$ (by the diagonal process, we obtain $\left\{n_{i}\right\}$ such that $\left\langle x_{k}^{*}, T^{n_{i}} x\right\rangle \rightarrow 0$ on a dense $\left.\left\{x_{k}^{*}\right\}\right)$, and $\left\{n_{i}\right\}$ even has density 1 .

2. The Proposition and the Theorem together improve the result of [2], where the assumption was $T^{n_{i}} x \rightarrow 0$ for some $\left\{n_{i}\right\}$ of density one (see following example). They also eliminate the use of the combinatorial machinery of [2].

3. The Proposition can also be proved by using the Banach-Mazur theorem, as in [3]. The simpler argument was suggested by Professor H. Furstenberg.

Lemma. Let $T$ be a positive contraction of $L_{1}$, having no fixed points (i.e., $T f=f \Rightarrow f=0$ ). If $g \in L_{1}$ and $\left\{n_{i}\right\}$ is an increasing sequence of density one such that $T^{n_{i}} g \rightarrow 0$ weakly, then $\left\|T^{n} g\right\|_{1} \rightarrow 0$.

Proof. Let $\left\{a_{i}\right\}$ be a bounded sequence of numbers, with $\left|a_{i}\right| \leqslant a$. If $\left\{n_{i}\right\}$ has density one (i.e., $n_{N} / N \rightarrow 1$ ), then

$$
\begin{aligned}
& \left|N^{-1} \sum_{i=1}^{N}\left(a_{i}-a_{n_{i}}\right)\right| \\
& \quad=\left|N^{-1} \sum_{i=1}^{N} a_{i}-N^{-1} \sum_{i=1}^{n_{N}} a_{i}+N^{-1} \sum\left\{a_{j}: j \notin\left\{n_{i}\right\}, j<n_{N}\right\}\right| \\
& \quad \leqslant 2\left(n_{N}-N\right) a / N \underset{N \rightarrow \infty}{\rightarrow} 0 .
\end{aligned}
$$


Hence, if $L$ is a Banach limit which preserves mean convergence [1, p. 33], $L\left\{a_{i}\right\}=L\left\{a_{n_{i}}\right\}$. The Lemma follows from the proof of Theorem 5.1 of Krengel and Sucheston [5], using that particular Banach limit.

EXAMPLE. Let $\mathcal{T}$ be an invertible weakly mixing transformation with no finite invariant measure, and $T$ the corresponding $L_{1}$ contraction (see Kakutani and Parry [4]). If $\int g=0$, then the conditions of our theorem are satisfied, and the Lemma shows that $T^{n_{i}} g$ does not converge weakly to 0 for $\left\{n_{i}\right\}$ of density one (because of $\left\|T^{-1}\right\|=1$ ).

PROBLEM. Is the condition in the Proposition necessary for the conditions of the Theorem? Since the conditions of the Theorem imply that 0 is a weak cluster point of $\left\{T^{n} x\right\}$, it is necessary if $\left\{T^{n} x\right\}$ is weakly sequentially compact, and it is also necessary when $X^{*}$ is separable.

In [6], R. Nagel studied a similar problem in the case that for every $x \in X,\left\{T^{n} x\right\}$ is weakly sequentially compact (see also [3]).

\section{REFERENCES}

1. S. R. Foguel, Ergodic theory of Markov processes, Van Nostrand Math. Studies, no. 21, Van Nostrand Reinhold, New York, 1969. MR 41 \#6299.

2. L. K. Jones, A mean ergodic theorem for weakly mixing operators, Advances in Math. 7(1971), 211-216. MR 44 \#2908.

3., A generalization of the mean ergodic theorem in Banach spaces, $\mathrm{Z}$. Wahrscheinlichkeitstheorie und Verw. Gebiete 27 (1973), 105-107.

4. S. Kakutani and W. Parry, Infinite measure preserving transformations with "mixing", Bull. Amer. Math. Soc. 69 (1963), 752-756. MR 27 \#3776.

5. U. Krengel and L. Sucheston, On mixing in infinite measure spaces, Z. Wahrscheinlichkeitstheorie Iund Verw. Gebiete 13 (1969), 150-164. MR 40 \#7424.

6. R. Nagel, Ergodic and mixing properties of linear operators, Proc. Roy. Irish Acad. Sect. A 74 (1974), 245-261.

Institut für Mathematische Statistik, 34, Göttingen, Germany

Department of Mathematics, Ohio State University, Columbus, Ohio 43210 